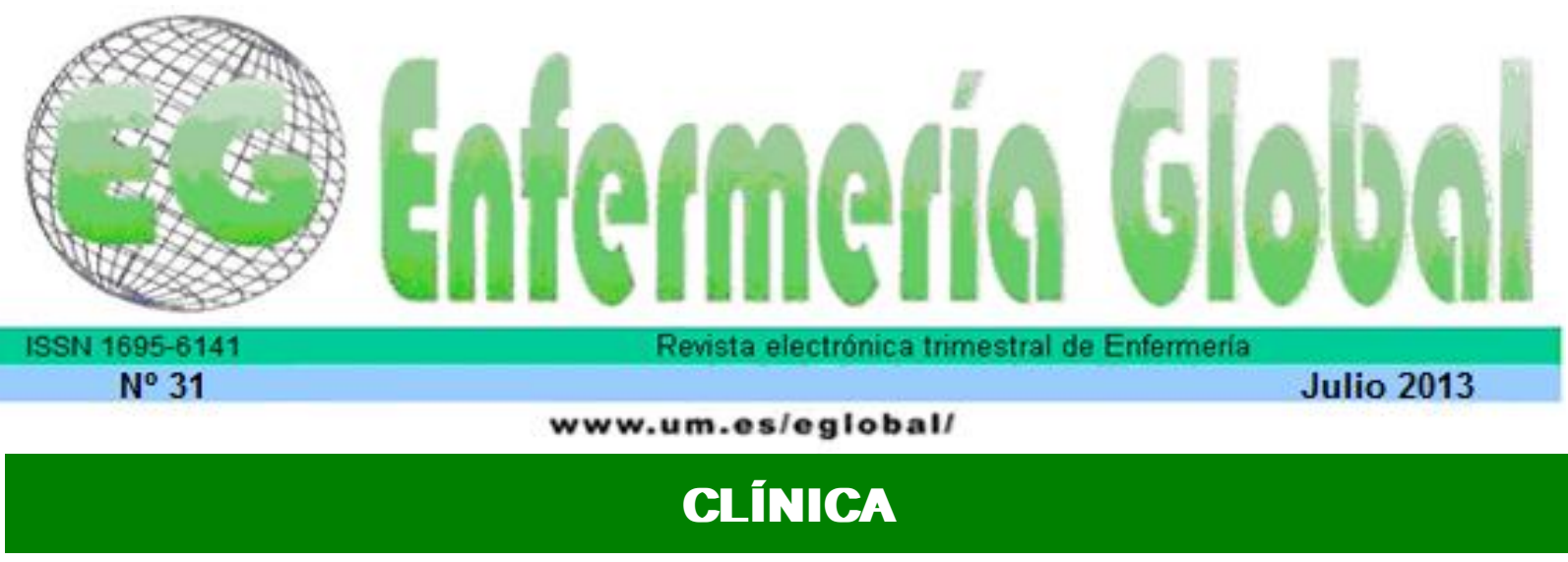

\title{
Complicaciones en pacientes sometidos a Angioplastia Coronaria Transluminal Percutánea
}

Complicações em pacientes submetidos à Angioplastia Coronariana Transluminal Percutânea Complications in patients undergoing Percutaneous Transluminal Coronary Angioplasty

\section{"Barbosa, Maria Helena **Moreira, Tassiana Márcia **Tavares, Jordânia Lumênia ${ }^{* * *}$ Andrade, Érica Vieira de ${ }^{* * *}$ Bitencourt, Marina Nolli ${ }^{* *}$ de Freitas, Kariny Beatriz Caiado ${ }^{* * * *}$ Cardoso, Gabriela Lucas}

*Doctora en Enfermería. Profesora del Departamento de Enfermería. E-mail: mhelena331@hotmail.com **Enfermera. " ${ }^{* * *}$ Enfermera. Máster en Atención a la Salud. *****Enfermera. Universidad Federal do Triângulo Mineiro (UFTM). Brasil.

Palabras clave: Angioplastia coronaria transluminal percutánea; complicaciones post-operatorias; cardiologia; enfermería.

Palavras-chave: Angioplastia transluminal percutânea coronária; complicações pós-operatórias; cardiologia; enfermagem

Keywords: Percutaneous transluminal coronary angioplasty; Postoperative complications; Cardiology; Nursing

\section{RESUMEN}

Esta investigación tuvo como objetivo identificar las complicaciones locales y sistémicas en pacientes sometidos a Angioplastia Coronaria Transluminal Percutánea y los factores de riesgo que pueden influir en la aparición de estas complicaciones. Estudio prospectivo, transversal, con análisis cuantitativo de los datos que fueron recolectados de 127 pacientes en la Unidad de Hemodinámica y en la Unidad de Cuidados Intensivos del hospital campo de estudio. Los datos fueron analizados mediante estadística descriptiva. Para investigar la asociación entre los factores de riesgo y las complicaciones después de la Angioplastia Coronaria Transluminal Percutánea se utilizó prueba de Chi cuadrado. Se observó que $79(62,2 \%)$ pacientes presentaron complicaciones. De éstos, $39(49,4 \%)$ tuvieron complicaciones locales, $25(31,7 \%)$, complicaciones sistémicas y $15(18,9 \%)$ habían asociado las complicaciones locales y sistémicas. Hubo una asociación estadísticamente significativa $(p<0,05)$ entre el uso continuo de los anticoagulantes después de la Angioplastia Coronaria Transluminal Percutánea y la presencia de complicaciones. Se encontró que la mayoría de los pacientes tuvo complicaciones y el uso de anticoagulante después del procedimiento está asociado con estas complicaciones. 


\section{RESUMO}

Esta pesquisa teve como objetivo identificar as complicações locais e sistêmicas em pacientes submetidos à Angioplastia Coronariana Transluminal Percutânea e os fatores de risco que podem influenciar na ocorrência destas complicações. Estudo prospectivo, transversal, com abordagem quantitativa dos dados que foram coletados de 127 pacientes na Unidade de Hemodinâmica e na Unidade de Terapia Intensiva do hospital campo de estudo. Os dados foram analisados segundo estatística descritiva. Para verificar a associação entre os fatores de risco e as complicações pós Angioplastia Coronariana Transluminal Percutânea foi utilizado o teste Qui-quadrado. Observou-se que $79(62,2 \%)$ pacientes apresentaram complicações. Destes, $39(49,4 \%)$ apresentaram complicações locais, 25 (31,7\%) complicações sistêmicas e 15 (18,9\%) apresentaram complicações locais e sistêmicas associadas. Verificou-se associação estatisticamente significativa $(p<0,05)$ entre o uso contínuo de anticoagulante após Angioplastia Coronariana Transluminal Percutânea e a presença de complicações. Evidenciou-se que a maioria dos pacientes apresentou complicações e o uso de anticoagulante após o procedimento está associado a estas complicações.

\section{ABSTRACT}

This research aimed to identify the local and systemic complications in patients undergoing Percutaneous Transluminal Coronary Angioplasty and risk factors that may influence the occurrence of these complications. It is a prospective cross-sectional study with a quantitative approach of the data that were collected from 127 patients in the Unit of Hemodynamic and in the Unit of Intensive Terapy of the hospital field of study. Data were analyzed using descriptive statistics. To investigate the association between risk factors and complications after Percutaneous Transluminal Coronary Angioplasty was used Chi-square test. It was observed that $79(62.2 \%)$ patients had complications. Of these, $39(49.4 \%)$ had local complications, 25 (31.7\%) systemic complications and $15(18.9 \%)$ had associated local and systemic complications. There was a statistically significant association $(p<0,05)$ between the continuous use of anticoagulants after Percutaneous Transluminal Coronary Angioplasty and presence of complications. It was evident that most of the patients had complications and use of anticoagulant after the procedure is associated with these complications.

\section{INTRODUCCIÓN}

Actualmente, las enfermedades cardiovasculares son la principal causa de muertes y, por tanto, un importante problema de salud pública dado que representa elevados costos en asistencia médica. De entre las enfermedades cardiovasculares, las coronarias y cerebrovasculares son responsables de los dos tercios de todos los óbitos ${ }^{(1)}$.

En los últimos años ha habido un importante crecimiento de la Cardiología Intervencionista, principalmente en lo que se refiere a las técnicas percutáneas de revascularización del miocardio. El perfeccionamiento del instrumental de angioplastia coronaria y la aparición de nuevos dispositivos, como los stents, han hecho posible el tratamiento de la gran mayoría de los pacientes ${ }^{(2-3)}$.

La angioplastia coronaria transluminal percutánea (ACTP) ha ido perfeccionándose desde su descripción inicial, a mediados de la década de los 60, y uno de los marcos más importantes en esa evolución fue su asociación con el uso de stents, que se mostraron tan importantes en el mantenimiento de los resultados inmediatos y a largo plazo ${ }^{(4-5)}$.

En un estudio que analizó el desempeño de la angioplastia transluminal percutánea coronaria primaria en Brasil de 1996 a 2000, reveló que en el periodo analizado, hubo aumento significativo de la realización de este procedimiento, en torno al $20 \%$, una media de $5 \%$ al año, en relación al total de intervenciones coronarias percutáneas 
computadas en el respectivo periodo ${ }^{(6)}$. Esta modalidad terapéutica presenta éxito indiscutible en el tratamiento de estenosis de arterias coronarias, renales y de la circulación arterial periférica ${ }^{(5)}$.

El acceso arterial femoral es la vía de elección más común, propiciando mayor rapidez y repetibilidad, fácil localización por el mayor calibre del vaso, variabilidad de materiales, exigiendo poca experiencia del operador. Consecuencia del avance tecnológico, surgen nuevos materiales y el desarrollo de vías arteriales alternativas como las vías transradial, braquial y ulnar han comenzado a ser utilizadas en los últimos años ${ }^{(7)}$.

Aunque la ACTP se considere un procedimiento seguro, sabemos que pueden ocurrir complicaciones y puede muchas veces estar asociada a factores relacionados tanto con las condiciones clínicas del cliente como las relacionadas con el procedimiento y materiales utilizados. Entre estas, las complicaciones vasculares han sido descritas con mayor frecuencia. Estas complicaciones normalmente están relacionadas con la calcificación de la arteria puncionada, obesidad, edad, sexo, hipertensión y el uso de anticoagulantes siendo el lugar de mayor incidencia de estas complicaciones el sitio de la punción en forma de hemorragias, sangrados, hematomas, fístulas, pseudoaneurismas e isquemias ${ }^{(7)}$.

Los pacientes sometidos a la ACTP pueden presentar complicaciones con repercusiones en su recuperación. Así, conocer las principales complicaciones y sus factores de riesgo ayudará a la elaboración de medidas que posibiliten intervenir precozmente y contribuir a la seguridad y calidad de la asistencia a estos pacientes.

En este contexto, el presente estudio tuvo como objetivo identificar las complicaciones locales y sistémicas en pacientes sometidos a la ACTP y los posibles factores de riesgo relacionados con el paciente y con el procedimiento que puede influir en la ocurrencia de estas complicaciones.

\section{MÉTODO}

Se trata de un estudio prospectivo, transversal, con enfoque cuantitativo de los datos, realizado en un hospital público de enseñanza que atiende pacientes de alta complejidad, localizado en el Municipio de Uberaba, Minas Gerais, Brasil.

Los datos fueron colectados por las investigadoras, en la Unidad de Hemodinámica y en la Unidad de Terapia Intensiva (UTI) de la referida institución campo de estudio, por un periodo de siete meses consecutivos (marzo a septiembre de 2009), tras la aprobación de este estudio por el Comitê de Ética en Investigación de la Universidad Federal do Triângulo Mineiro bajo parecer nํㅜ 1266 .

La colecta de los datos fue realizada por medio de evaluación del paciente, utilizándose un instrumento específico para este fin, que consta de tres partes. La primera se refería a los datos clínicos y demográficos del paciente; la segunda, a los aspectos relacionados con el procedimiento de ACTP y la tercera, a la evaluación de los parámetros vitales tras la ACTP y a la intensidad del dolor en el lugar de la punción arterial. 
Entre los parámetros vitales evaluados, se utilizaron los siguientes valores de referencia: $\mathrm{FC}=<100 \mathrm{bpm}^{(8)} ; \mathrm{FR}=<20 \mathrm{irpm}^{(8)}$; $\mathrm{T}=35,5$ a $37^{\circ} \mathrm{C}$ con media de 36,0 a $36,5^{\circ} \mathrm{C}^{(9)}$, PAS $<130 \mathrm{mmHge} \mathrm{PAD}<85 \mathrm{mmHg}^{(10)}$.

La intensidad del dolor en el lugar de la punción fue evaluada por medio de la escala numérica verbal graduada de 0 a 10, donde 0 significa ausencia de dolor y 10 el peor dolor que ha sentido alguna vez. La intensidad del dolor fue así clasificada en sin dolor (0), dolor leve (1 a 4), dolor moderado (5 a 7) y dolor intenso (8 a 10) ${ }^{(11)}$.

La evaluación de los pacientes se realizó al término del procedimiento de ACTP, inmediatamente después de la retirada del introductor de la vía arterial y antes del alta de la UTI, considerando que en el hospital, campo de estudio, los pacientes permanecen en la UTI hasta 24 horas tras la realización de la ACTP.

Se incluyeron en esta investigación 127 pacientes adultos con edad igual o superior a 18 años, después de la aceptación en participar de la pesquisa y de la firma del Término de Consentimiento Libre y Esclarecido. Se excluyeron los pacientes que presentaban comorbidades graves asociadas y aquellos con inestabilidad hemodinámica al ser admitidos en la unidad para la realización del procedimiento.

Los datos fueron introducidos en un banco de datos electrónico del programa Excel $X P \AA$ de Microsoft ${ }^{\circledR}$ y exportados al software Statistical Package for the Social Sciences para procesamiento y análisis. Se utilizó estadística descriptiva con análisis univariado de los datos. Para verificar si hubo asociación entre las complicaciones tras ACTP y los posibles factores de riesgo se utilizó el test Qui-quadrado. Los resultados fueon considerados significativos en nivel de significancia de $5 \%(p<0,05)$.

\section{RESULTADOS}

En el periodo de marzo a septiembre de 2009, 185 pacientes fueron sometidos a la ACTP, de los cuales 127 (68,6\%) atendían a los criterios de inclusión y constituyeron la muestra de este estudio.

La media de edad de los pacientes fue de 60,8 años con variación de 30 a 84 años; $83(65,4 \%)$ pacientes eran del sexo masculino; 46 (36,2\%) eran jubilados y el Índice de Masa Corporal (IMC) medio fue de $26,0 \mathrm{Kg} / \mathrm{m}^{2}$ con variación de 13,0 a $38,0 \mathrm{Kg} / \mathrm{m}^{2}$.

En cuanto a las comorbidades, $71(55,0 \%)$ pacientes presentaban hipertensión arterial sistémica (Figura 1). 


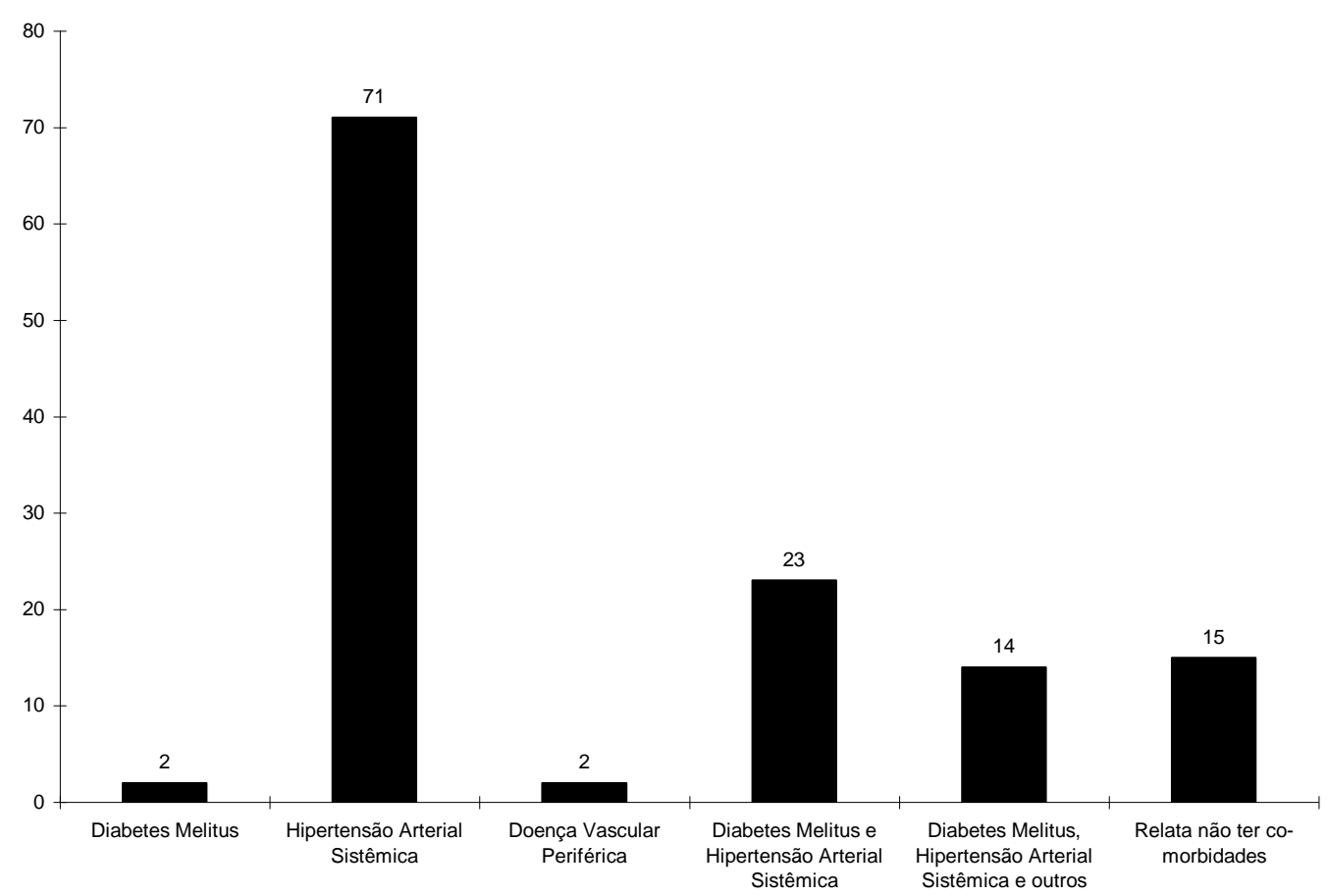

Figura 1- Distribución de los pacientes según el porcentaje de comorbidades. Uberaba (MG), Brasil, 2009.

Se observó que 44 (34,6\%) pacientes fumaban, $13(10,2 \%)$ consumían bebidas alcohólicas; 5 (3,9\%) consumían drogas ilícitas, 22 (17,3\%) fumaban y consumían bebida alcohólica, $4(3,1 \%)$ consumían tabaco, alcohol y drogas ilícitas y $39(30,9 \%)$ relataron no hacer uso de estas substancias.

En relación a los lugares de punción arterial para el procedimiento de ACTP, se observó que en $124(97,6 \%)$ pacientes se utilizó la vía femoral y en $3(2,4 \%)$ la vía transradial. El tiempo medio de compresión del lugar, tras la retirada del introductor, fue de 38,9 minutos, con variación de 15 a 90 minutos.

De los 127 pacientes evaluados, 79 (62,2\%) presentaron complicaciones tras la realización de la ACTP. De estos, 39 (49,4\%) presentaron complicaciones locales (sangrado, hematoma, equimosis), 25 (31,7\%) complicaciones sistémicas (retención urinaria, alteración del ritmo cardiaco) y 15 (18,9\%) presentaron complicaciones locales y sistémicas asociadas. El número medio de complicaciones por paciente fue de 1,1 (Tabela I).

Tabla I- Distribución de los pacientes en cuanto a los tipos de complicacionees locales y sistémicas. Uberaba (MG), Brasil, 2009.

\begin{tabular}{lcclcc}
\hline $\begin{array}{c}\text { Complicaciones } \\
\text { locales }\end{array}$ & $\mathbf{N}$ & $\%$ & Complicaciones sistémicas & $\mathbf{N}$ & $\%$ \\
\hline Sangrado & 13 & 10,2 & Retención urinaria & 12 & 9,4 \\
Hematoma & 29 & 22,8 & Alteración del ritmo cardiaco & 8 & 5,5 \\
Equimosis & 6 & 4,7 & $\begin{array}{l}\text { Retención urinaria y Alteración del } \\
\text { ritmo cardiaco }\end{array}$ & 17 & 13,4 \\
Hematoma y & 5 & 4,0 & Otros & 2 & 1,6 \\
Equimosis & 74 & 58,3 & No presenta & 88 & 70,1 \\
No presenta & 127 & 100,0 & TOTAL & 127 & 100,0 \\
\hline TOTAL & & & & & \\
\hline
\end{tabular}


En relación a los parámetros vitales verificados, los valores medios fueron de 120,8 $\mathrm{mmHg}$ para presión arterial sistólica; $72,9 \mathrm{mmHg}$ para presión arterial diastólica; 71,6 bpm para frecuencia cardiaca, $20,2 \mathrm{rpm}$ para frecuencia respiratoria y $36,0^{\circ} \mathrm{C}$ para temperatura.

En cuanto a la evaluación del dolor, $67(52,7 \%)$ pacientes relataron presentar dolor en el lugar de la punción arterial. De estos, $35(52,2 \%)$ pacientes refirieron dolor de poca intensidad, $16(23,9 \%)$ de intensidad moderada y $16(23,9 \%)$ refirieron dolor de fuerte intensidad.

En cuanto a los posibles factores de riesgo relacionados con el paciente (edad, sexo y comorbidades), no hubo asociación estadísticamente significativa con la ocurrencia de complicaciones tras el ACTP. Entre los posibles factores de riesgo relacionados con el procedimiento (tiempo de compresión, número de procedimientos realizados y uso de terapéutica anticoagulante), se verificó que hubo asociación estadísticamente significativa $(p=0,01)$ con el uso continuo de anticoagulante tras el procedimiento de ACTP (Tabela II).

Tabla II- Factores de riesgo que podrían influir en la ocurrencia de las complicaciones. Uberaba (MG), Brasil, 2009.

\begin{tabular}{|c|c|c|c|}
\hline \multicolumn{4}{|c|}{ Complicaciones } \\
\hline & Sí & No & $p$ (valor) \\
\hline \multicolumn{4}{|l|}{ Edad } \\
\hline Menos de 60 años & 41 & 22 & \multirow{2}{*}{0,507} \\
\hline Más de 60 años & 38 & 26 & \\
\hline \multicolumn{4}{|l|}{ Género } \\
\hline Masculino & 50 & 33 & \multirow{2}{*}{0,531} \\
\hline Femenino & 29 & 15 & \\
\hline \multicolumn{4}{|l|}{ Comorbidades } \\
\hline Sí & 11 & 4 & \multirow{2}{*}{0,344} \\
\hline No & 68 & 44 & \\
\hline \multicolumn{4}{|l|}{ Tiempo de compresión } \\
\hline Menos de 30 minutos & 17 & 5 & \multirow{2}{*}{0,253} \\
\hline 30 minutos o más & 60 & 33 & \\
\hline \multicolumn{4}{|l|}{$\begin{array}{l}\text { Número de procedimientos } \\
\text { anteriores }\end{array}$} \\
\hline Ninguno & 46 & 30 & \multirow{3}{*}{0,206} \\
\hline De 1 a 2 & 28 & 18 & \\
\hline Más de 2 & 5 & 0 & \\
\hline \multicolumn{4}{|l|}{$\begin{array}{l}\text { Uso de anticoagulante antes } \\
\text { del procedimiento }\end{array}$} \\
\hline Sí & 18 & 20 & \multirow{2}{*}{0,078} \\
\hline No & 49 & 23 & \\
\hline \multicolumn{4}{|l|}{$\begin{array}{l}\text { Uso de antiagregante } \\
\text { plaquetario antes del } \\
\text { procedimiento }\end{array}$} \\
\hline Sí & 18 & 14 & \multirow[b]{2}{*}{0,605} \\
\hline No & 49 & 29 & \\
\hline
\end{tabular}


Uso de anticoagulante tras

el procedimiento

\begin{tabular}{|c|c|c|c|}
\hline $\begin{array}{l}\text { Sí } \\
\text { No }\end{array}$ & $\begin{array}{l}56 \\
23\end{array}$ & $\begin{array}{l}23 \\
25\end{array}$ & 0,010 \\
\hline $\begin{array}{l}\text { Uso de } \\
\text { plaquetario } \\
\text { procedimiento }\end{array}$ & $\begin{array}{c}\text { antiagregante } \\
\text { tras el }\end{array}$ & & \\
\hline $\begin{array}{l}\text { Sí } \\
\text { No }\end{array}$ & $\begin{array}{l}61 \\
18\end{array}$ & $\begin{array}{l}37 \\
11\end{array}$ & 0,986 \\
\hline
\end{tabular}

En todos los pacientes evaluados en este estudio, el procedimiento de ACTP ocurrió con colocación de stent. El número medio de stents implantados fue de 1,4 por paciente, con variación de 1 a 3 stents.

\section{DISCUSIÓN}

La ACTP se ha convertido en un procedimiento utilizado mundialmente y el conocimiento de factores de riesgo/predictores de complicaciones puede asegurar la obtención de mejores resultados a corto y largo plazo ${ }^{(10)}$.

Se observó en el presente estudio mayor prevalencia de complicaciones en pacientes del género masculino y con sobrepeso. Esto tal vez pueda estar asociado a diferencias relacionadas con aspectos biológicos, culturales y estilo de vida. La explicación biológica sería la protección femenina del estrógeno, que influye en el sistema circulatorio, promueve vasodilatación e inhibe la progresión de procesos arteroscleróticos evitando procesos isquémicos ${ }^{(12-13)}$.

Por ello, estos resultados contradicen, en parte, otros estudios ${ }^{(7,12)}$ en los que pacientes también con sobrepeso $\left(\mathrm{IMC}>25 \mathrm{Kg} / \mathrm{m}^{2}\right)$, del género femenino, tuvieron mayor prevalencia de complicaciones.

La población del presente estudio se encuentra en una franja de edad media de 60,8 años en que hay prevalencia de enfermedades coronarias, lo que puede estar relacionado con el hecho de que la hipertensión arterial sistémica haya sido la principal comorbidad (55,0\%) evidenciada, tanto aislada cono asociada a la diabetes mellitus. Además, el aumento de la longevidad posibilita periodos más largos de exposición a los factores de riesgo, resultando en una mayor probabilidad de manifestación clínica de estas dolencias ${ }^{(1)}$.

Con relación a la vía escogida para la punción, en este estudio prevaleció el abordaje arterial femoral en $97,6 \%$ de los pacientes, lo que corrobora con otros estudios de metodología semejante ${ }^{(14-15)}$. A pesar de ser la vía femoral la más frecuente, los procedimientos realizados por vía radial obtienen mayor éxito, menor ocurrencia de eventos cardíacos adversos, óbito y complicaciones vasculares ${ }^{(14-15)}$. Otros autores también señalan que la diversidad de las vías arteriales de acceso es eficaz en cardiología intervencionista ${ }^{(3)}$.

A pesar de que la ACTP sea un procedimiento seguro, pueden ocurrir complicaciones $^{(7)}$. Entre los posibles factores de riesgo para ocurrencia de complicaciones tras ACTP, algunos autores ${ }^{(16)}$ señalan el uso de anticoagulantes antes del procedimiento, sexo femenino, presión arterial sistólica mayor de 160 $\mathrm{mmHg}$, número de veces en que la arteria fue puncionada y cambio de profesional 
durante la compresión como algunos de los factores que presentan asociación estadísticamente significativa con la ocurrencia de complicaciones. Estos resultados contraponen los resultados del presente estudio, en el cual solamente el uso continuo de anticoagulante tras el procedimiento de ACTP tuvo significancia estadística, aunque no todos los factores descritos han sido investigados.

Los resultados de investigación realizada en el Instituto de Cardiología de Rio Grande do Sul ${ }^{(17)}$ también divergen de estos resultados. Los investigadores evidenciaron que entre los potenciales predictores de complicaciones, solo el calibre del introductor alcanzó significancia estadística, demostrando relación directa entre calibre del introductor y complicaciones vasculares. Otros investigadores ${ }^{(15)}$ también indicaron que la utilización de catéteres de menor calibre disminuye la incidencia de complicaciones en este tipo de procedimiento.

En cuanto a las complicaciones sistémicas, la retención urinaria es la principal complicación pos-procedimiento por via femoral, siendo que algunos pacientes necesitan de intervención como cateterismo vesical por no responder a medidas no invasivas de diuresis espontánea ${ }^{(18)}$.

En otro estudio ${ }^{(7)}$ se observó que entre los procedimientos de ACTP realizados por vía femoral, poco más del $25 \%$ de los pacientes necesitaron de cateterismo vesical, y cuando se utilizó la vía radial, menos del 3\% de los pacientes presentaron retención urinaria.

En este estudio, la retención urinaria y la alteración del ritmo cardiaco fueron las principales complicaciones sistémicas evidenciadas. Esto tal vez pueda estar relacionado con el hecho de que los pacientes que realizan el procedimiento por vía femoral quedan en cama por algunas horas dependiendo de la evolución clínica, al contrario de lo que sucede cuando es utilizada la vía radial, en que el paciente es libre de moverse en la cama y caminar muy pronto ${ }^{(19)}$.

Otro aspecto evaluado fue la intensidad del dolor en el lugar de la punción arterial, mostrando que la mayoría de los pacientes refirió dolor de poca intensidad. Este resultado diverge de otra investigación en la que el $38 \%$ de los pacientes refirieron dolor moderado cuando el procedimiento fue realizado por abordaje femoral y $35 \%$ refirieron dolor de poca intensidad por vía radial ${ }^{(7)}$.

\section{CONCLUSIÓN}

En este estudio se identificaron las siguientes complicaciones locales: equimosis, hematomas y sangrado. La retención urinaria y la alteración del ritmo cardiaco fueron las principales complicaciones sistemicas después del procedimiento de ACTP. La hipertensión arterial sistémica fue identificada como la principal comorbidad en los pacientes estudiados. En cuanto a la vía de acceso, prevaleció el abordaje femoral. Entre los posibles factores de riesgo para la ocurrencia de complicaciones, solamente el uso continuo de anticoagulante tras la ACTP presentó asociación estadísticamente significativa $(p<0,05)$.

Los resultados de esta investigación contribuirán a la elaboración de metas para reducir la ocurrencia de complicaciones tras ACTP, así como al desarrollo de protocolos asistenciales. 
Este estudio presenta como limitación la no evaluación del número del calibre de los introductores, lo que no interfirió en los resultados obtenidos. Se sugiere la realización de nuevos estudios que incluyan el abordaje de esta temática.

\section{REFERENCIAS}

1. Barreto SM, Passos VMA, Cardoso ARA, Costa MFL. Quantificando o risco de doença coronariana na comunidade: Projeto Bambuí. Arq bras cardiol. 2003 dez; 81(6):549-55.

2. Osterne ECV, Alexim GA, Motta VP, Lins RMM, Carvalho EF, Carneiro MV et al. Angioplastia coronariana com suporte circulatório por balão intra-aórtico: experiência inicial.Arq bras cardiol. 1999 ago; 73(2):191-95.

3. Gus I, Fischmann A, Medina C. Prevalência dos fatores de risco da doença arterial coronariana no Estado do Rio Grande do Sul. Arq bras cardiol. 2002 maio; 78(5):47883.

4. Pereira $\mathrm{AH}$. Angioplastia da carótida versus endarterectomia: o velho e o novo. J vasc bras. 2006 set; 5(3):169-73.

5. Piske RL, Ferreira MS, Campos CMS, Teixeira JAN, Piske LEB, Fonseca NC et al.Técnica de proteção cerebral na angioplastia e "stent" de carótida:um procedimento eficaz contra embolia. Arq Neuro-Psiquiatr. 2003 jun; 61(2):296-302.

6. Mattos LA, Souza AP, Ibraim MF, Campos Neto CM, Labrunie A, Alves CR, Saad J. Evolução temporal com a utilização da angioplastia coronariana primária no infarto agudo do miocárdio no Brasil: análise dos preditores de sucesso e dos eventos adversos hospitalares em 9.434 pacientes. Arq bras cardiol. 2002 out; 79(4):405-18. 7. Armendaris MK, Azzolin KO, Alves FJMS, Ritter SG, Moraes MAP. Incidence of vascular complications in patients submitted to percutaneous transluminal coronary angioplasty by transradial and transfemoral arterial approach. Acta Paul Enferm. 2008 jan/mar; 21(1):107-11.

8. Barros E, Albuquerque GC, Pinheiro CTS, Czepielewski MA. Exame clínico: consulta rápida. $2^{a}$ ed. Porto Alegre: Artmed; 2004.

9. Porto CC, Guimarães EMB, AlmeidaNeto JC. Exame físico geral. In:

Semiologia Médica. 2ª ed. Rio de Janeiro: Guanabara Koogan; 1994.

10. Sociedade Brasileira de Cardiologia. VI Diretrizes Brasileiras de Hipertensão. Rev bras Hipertens. 2010 jan/mar; 17(1):1-64.

11. Calil AM, Pimenta CAM. Intensidade da dor e adequação de analgesia. Rev Latinoam Enferm, 2005 set/out;13(5):692-99.

12. Brito JR, Magalhães MA, Nascimento TCDC, Amorim IMG, Almeida BO, Abizaidi A et al. Incidência e preditores contemporâneos de complicações vasculares após intervenção coronária percutânea. Rev bras cardiol invasiva, 2007 out/dez; 15(4):39499.

13. Ceravolo GS, Tostes RC, Fortes ZB, Carvalho MHC. Efeitos do estrógeno no sistema cardiovascular. Hipertensão. 2007; 10(4):124-30.

14. Pereira $A H$, Grudtner MA. Angioplastia transluminal percutânea e stents endovasculares. Angiologia e cirurgia vascular: guia ilustrado. Maceió: Uncisal/Ecmal \& Lava; 2003.

15. Dall'Orto CC, Lapa GA, Carneiro Neto JD, Carnieto NM, Oliveira Neto JB, Mauro MFZ et al. Angioplastia coronária nas indicações off-label: comparação das vias radial vs. femoral. Rev bras cardiol invasive. 2010 jun; 18(2):177-84.

16. Andersen K, Bregendahl M, Kaestel H, Skriver M, Ravkilde J. Haematoma after coronary angiography and percutaneous coronary intervention via the femoral artery frequency and risk factors. Eur J Cardiovasc Nurs. 2005 Jun; 4(2):123-27. 
17. Zanatta LG, Cardoso CO, Mota FM, Conti EP, Diehl D, Rodrigues APR et al. Preditores e incidência de complicações vasculares após a realização de intervenções coronárias percutâneas: achados do registro IC-FUC. Rev bras cardiol invasiva. 2008 jul/set; 16(3):301-06.

18. Augustin AC, Quadros AS, Yordi LM, Sarmento LR. Retenção urinária e seu manejo após intervenções coronarianas percutâneas. Tema livre do 12nd Congresso da Sociedad Latinoamericana de Cardiología Intervencionista; 28th Congresso da Sociedade Brasileira de Hemodinânica e Cardiologia Intervencionista; 11th Jornada de Enfermagem, 2006, Recife. Rev bras cardiol invasiva. 2006; 14(2):247.

19. Tift Mann J, Cubeddu G, Schneider J, Arrowood M. Right radial access for PTCA: a prospective study demonstrates reduced complications and hospital charges. $J$ invas cardiol. 1996; 8 (suppl D):40D-44. 